BULLETIN Bulletin hispanique

HISPANIQUE Université Michel de Montaigne Bordeaux

123-2 | 2021

Investigaciones semánticas y léxicas actuales

\title{
Blandine Daguerre, Passage et écriture de l'entre-deux dans El Pasajero de Cristóbal Suárez de Figueroa
}

Pessac : PUPPA, collection PrimaLun@ 3, 2020

Fabrice Quero

\section{OpenEdition}

Journals

Édition électronique

URL : https://journals.openedition.org/bulletinhispanique/14554

DOI : 10.4000/bulletinhispanique. 14554

ISBN : $1775-3821$

ISSN : 1775-3821

Éditeur

Presses universitaires de Bordeaux

Édition imprimée

Date de publication : 20 décembre 2021

Pagination : 381-384

ISBN : 979-10-300-0-745-9

ISSN : 0007-4640

Référence électronique

Fabrice Quero, «Blandine Daguerre, Passage et écriture de l'entre-deux dans El Pasajero de Cristóbal Suárez de Figueroa », Bulletin hispanique [En ligne], 123-2 | 2021, mis en ligne le 20 décembre 2021, consulté le 05 février 2022. URL : http://journals.openedition.org/bulletinhispanique/14554; DOI : https://doi.org/10.4000/bulletinhispanique.14554

Ce document a été généré automatiquement le 5 février 2022.

Tous droits réservés 


\section{Blandine Daguerre, Passage et écriture de l'entre-deux dans El Pasajero de Cristóbal Suárez de Figueroa}

Pessac : PUPPA, collection PrimaLun@ 3, 2020

Fabrice Quero

\section{RÉFÉRENCE}

Daguerre, Blandine, Passage et écriture de l'entre-deux dans El Pasajero de Cristóbal Suárez de Figueroa, Pessac, PUPPA, collection PrimaLun@ 3, 2020, 312 p., [en ligne] https://unaeditions.fr/passage-et-ecriture-de-lentre-deux-dans-el-pasajero-de-cristobal-suarezde-figueroa

L'ouvrage électronique publié par Blandine Daguerre est une version remaniée de la thèse de doctorat soutenue en 2017 devant l'Université de Pau et des Pays de l'Adour. Il est consacré à un texte remarquablement complexe : le dialogue intitulé El Pasajero et publié par Cristóbal Suárez de Figueroa en 1617. L'ouvrage de 315 pages est dense. L'architecture, classique, repose sur un plan équilibré où trois parties d'égale longueur sont elles-mêmes subdivisées en trois sous-parties. Elles sont suivies d'annexes qui contiennent différents tableaux synoptiques intéressant le texte qui est l'objet principal de l'étude (répartition des compositions poétiques qui jalonnent le dialogue ; variations génériques; niveaux narratifs et niveaux dialogiques du récit de Juan) et, de façon plus générale, des aspects et thématiques qui traversent toute l'œuvre de Suárez de Figueroa (citations de El Pasajero et de Pusilipo où le sème de la conversation apparaît; autorités communes aux trois dernières œuvres de Figueroa). On y trouve également trois captations audiovisuelles accessibles à partir d'un $\mathrm{QR}$ code: deux adaptations théâtrales d'un passage du Quichotte et du Buscón; une interview d'Antonio Campos à propos de la seconde. Quoique la théâtralité du dialogue de Figueroa soit envisagée çà et là dans l'étude, ce parti pris est moins convaincant. L'ouvrage se 
referme sur une bibliographie divisée en trois sections : une première contient de nombreuses sources primaires, soit des textes contemporains de l'œuvre de Suárez de Figueroa et, plus largement, des œuvres qui résonnent dans El Pasajero; une deuxième réunit, sous l'étiquette "sources secondaires", les références critiques utilisées; la dernière dresse la liste des divers ouvrages et sites généraux auxquels l'auteur a eu recours. Il s'agit là d'un vaste ensemble de références qui est à l'aune de la richesse du dialogue que Blandine Daguerre étudie et de l'approche méthodologique choisie pour ce faire.

2 Comme le titre de l'ouvrage l'annonce et comme le corroborent les nombreuses occurrences du terme tout au long du texte, l'entre-deux est le centre gravité de l'ensemble de la démonstration. Dans la continuité des travaux de Lucien Dallenbach sur la spécularité et la mise en abyme, cette notion, empruntée à Nadine Ly, est mobilisée dès l'introduction pour inscrire le dialogue de Suárez de Figueroa dans une poétique du discontinu qui est, de l'avis de l'hispaniste, un des principaux traits distinctifs de la production littéraire du Siècle d'or. Blandine Daguerre reprend à son compte cette affirmation afin de proposer une clé de lecture grâce à laquelle aller audelà du foisonnement thématique, intertextuel - où les auto-citations abondent dans une démarche de réécriture -, et des emprunts génériques multiples d'un dialogue éminemment hybride. L'entre-deux tient lieux de «boussole » afin de guider le lecteur moderne, au-delà d'un apparent désordre, dans les méandres d'une économie faite de va-et-vient, imposés par les diverses manifestations du discontinu, et porteuse de discours qui attestent le passage ou le basculement, esthétique et idéologique, d'une époque à une autre. Ce faisant, l'ouvrage cible un point aveugle de la critique et prend le contre-pied de diverses études sur El Pasajero. Ainsi, le dialogue de Suárez de Figueroa est étudié pour lui-même et sous l'ensemble de ses aspects, après des travaux, précieux pour certains, qui ont mis en lumière tel dimension du texte ou telle particularité de la biographie ou de la personnalité de l'auteur. En somme, Blandine Daguerre ne craint pas d'interroger tout ce que la critique a tenu pour acquis jusqu'à présent.

3 La première partie de l'étude revient sur l'inscription de l'œuvre dans la seule catégorie du dialogue en raison du fort degré d'hybridité qui y est observable. Plusieurs éléments de contextualisation (dans l'ensemble de la production de Suárez de Figueroa, par rapport aux œuvres de ses devanciers et de ses contemporains, au sein des courants esthétiques et idéologiques vivaces en la période charnière où l'auteur écrit) sont offerts au lecteur afin de dégager la singularité de El Pasajero. Le dialogue répond aux principales caractéristiques du genre. Cependant, l'enjeu de la démonstration est ailleurs. En affirmant qu'il est «un dialogue sui generis », Blandine Daguerre entend dégager l'œuvre de ce cadre générique. Une utilisation plus systématique de la littérature sur la question - nous pensons notamment aux travaux d'Ana Vian Herrero - aurait sans doute permis d'apporter quelques nuances à ce propos sur un texte qui demeure ancré, à certains égards, dans une tradition toute renaissante. Mais l'ambition affichée ici est, avant tout, de donner à voir la complexité des phénomènes transtextuels (notamment avec des sources italiennes) et transgénériques qui traversent les échanges entre les interlocuteurs mais également les sections narratives. La question des relations entre dialogue et miscellanées, auxquelles est redevable une poétique de la varietas, est posée de manière frontale et vient renforcer l'analyse lexicosémantique - cette méthodologie, qui est complétée parfois par des analyses étymologiques, est souvent mise en œuvre - du titre de l'ouvrage de Figueroa. C'est le 
texte lui-même, véritable laboratoire littéraire, qui est pasajero et qui installe les discours multiples qu'on y entend dans un savant entre-deux, formel et idéologique. Les pages consacrées à la «fictionnalisation de l'auteur » où le personnage du Doctor, comme figure de projection de l'auteur, est étudié, confirment cette conclusion. Comme l'observe Blandine Daguerre, l'expérimentation voisine ici avec la critique.

4 La deuxième partie de Passage et écriture de l'entre-deux dans El Pasajero de Cristóbal Suárez de Figueroa met les conclusions de la précédente à l'épreuve d'une analyse méticuleuse de la chronologie, des espaces et des interlocuteurs du dialogue et des personnages apparaissant dans les récits interpolés. La méthodologie lexicosémantique, et onomastique, se voit complétée par une approche narratologique. Dans le traitement du temps et de l'espace, Suárez de Figueroa joue de l'entre-deux en articulant espaces topiques (le locus amœnus du roman pastoral, par exemple) et indicateurs de temporalité destinés à doter les interlocuteurs d'une véritable épaisseur. Chacun possède un itinéraire personnel où se rencontrent le déterminisme de la théorie des humeurs exposée par Huarte de San Juan au siècle précédent et l'effet de perspective produit par la référence à un passé amendé. L'analyse des récits " autobiographiques ", autrement dit où chacun des interlocuteurs se raconte, ainsi que des blocs analeptiques montre, une nouvelle fois, la complexité de l'économie du dialogue. Les protagonistes des récits interpolés répondent quant à eux, comme il est d'usage dans ces passages narratifs intercalés, à une typologique traditionnelle (soldat, duègne, pícaro). Blandine Daguerre montre que ces personnages sont au service d'une dénonciation des travers de la société de l'époque qui parcourt tout El Pasajero. Interlocuteurs et personnages contribuent ainsi, par réfraction, à la reconstitution d'une société en crise et en proie au doute à la fin du règne du Philippe III. Les excursus moraux qui émaillent le dialogue et la présence de diverses thématiques typiquement baroques (faux-semblants, masques, déguisements) donnent à penser que cet entredeux est également la marque d'une forme de versatilité, voire d'instabilité.

Ces développements conduisent de façon naturelle à interroger à nouveaux frais, dans une dernière partie, la poétique de l'entre-deux. L'ouvrage de Blandine Daguerre fait ici retour sur plusieurs aspects du texte de Suárez de Figueroa mis en relief dans les pages précédentes. Elle les étudie afin de révéler la structuration réticulaire du «tissu » du dialogue et de mettre au jour une véritable fabrique du discontinu. On laisse entrevoir le savant agencement des digressions et des détours, véritablement fondateurs de l'économie de l'œuvre et naturellement au service de la multiplicité des thématiques qu'elle contient et de la polyphonie énonciative qui les porte. L'auteur montre qu'en disjoignant à dessein ce qui est connexe et en substituant à la linéarité du propos des reprises thématiques, lexicales ou narratives, dans des mouvements d'oscillation, El Pasajero porte un regard critique sur la société de son temps. La question du mérite, unique vecteur d'unité au sein de l'œuvre, et celle de la justice, son corollaire, est en débat d'un bout à l'autre du dialogue. Sous la plume de Suárez de Figueroa, elle ne ressortit pas uniquement aux armes et à la noblesse, mais irradie également le champ de la littérature. À cet égard, les nombreux glissements entre les différentes instances énonciatives participent d'une entreprise générale de brouillage, voire d'abolition des frontières textuelles et de débordement de la fiction proprement dite sur la non-fiction.

Entre micro-analyses et visions surplombantes du texte de Suárez de Figueroa, Blandine Daguerre s'attache à mettre au jour la cohérence d'un texte complexe, au riche hypotexte, dans le désordre qu'il organise savamment. Reflet d'une époque 
charnière, El Pasajero offre, avec toutes les difficultés qu'une telle entreprise suppose, une reconfiguration d'un système en crise ou parvenu à un point de bascule à tout le moins. Dans une étude minutieuse et exigeante, Blandine Daguerre parvient à montrer la fécondité poétique de la notion d'entre-deux qui vertèbre sa démonstration. Cet ouvrage constitue, à n'en pas douter, un travail éminemment suggestif pour les études à venir sur la production de cet auteur.

\section{AUTEURS}

\section{FABRICE QUERO}

Université Paul-Valéry Montpellier 3 